\title{
Los espacios virtuales educativos en el ámbito de Internet: un refuerzo a la formación tradicional
}

\author{
Francisco José García \\ Peñalvo \\ fgarcia@usal.es \\ Universidad de Salamanca \\ Joaquín García \\ Carrasco \\ carrasco@usal.es
}

\section{Introducción}

El auge de Internet en general, y de los servicios web en particular, ha traído consigo la aparición de espacios de trabajo virtuales diversos como metáforas de interacción entre personas pertenecientes a distintos colectivos profesionales que, aun estando diseminados geográficamente, tienen un lugar común de intercambio de información e intereses en dichos espacios virtuales, siendo canalizada la entrada a éstos mediante los tan popularizados portales de Internet. Ejemplo claro de esta virtualización de la vida cotidiana es el aumento de interés que socialmente están despertando las denominadas "empresas.com" y, en general, el comercio electrónico; el crecimiento exponencial de páginas web en las que instituciones y personas aportan información sobre procesos de actividad, recursos para la actividad, conocimientos de todo tipo... ampliando el espacio de comunicación colectiva más allá de los límites técnicos, económicos o de distribución que la comunicación escrita permitían. La red constituye hoy el acumulador de experiencia y de conocimiento más imponente que jamás haya existido. Se potencia así la capacidad de memoria, función imprescindible para la actividad en la Zona de Construcción del Conocimiento y de configuración de la acción.

La enseñanza no es, y no debe ser, ajena a las posibilidades que abren las denominadas nuevas tecnologías. Los mecanismos primarios de la formación siguen estando, para todos los sujetos, en el mundo de la vida, para el sujeto particular y para la humanidad en su conjunto. La combinación de los servicios web con las capacidades hipermedia y multimedia debe ser canalizada a través de adecuadas interfaces de usuario, capaces de soportar toda esta potencia formativa, sin abrumar ni desbordar al discente.

La perspectiva que desde este artículo se desea transmitir, y por ende desde el grupo de trabajo e investigación de los autores, es que los espacios virtuales educativos no van encaminados a terminar con las actividades formativas presenciales tradicionales, sino que deben ser complementarios a éstas, permitiendo una mejora sustancial de la calidad tanto del canal de comunicación entre profesores y alumnos, como en los materiales docentes utilizados en el proceso educativo; todo ello sin descartar la apertura de nuevos caminos de formación mediante experiencias controladas de educación a distancia.

La defensa del mantenimiento de los medios educativos presenciales se justifica en que la condición de ser humano o ser inteligente se debe de forma fundamental al contacto con otros seres humanos e inteligentes. De hecho, Aristóteles, hace ya más de 2000 años, definió al hombre como un animal social por naturaleza. Esto se refleja en el proceso de formación de los niños, donde la autoconciencia que les permite definirse como seres individuales sólo la adquieren en contacto con los demás. Cabe señalar, incluso, que al hombre le sería imposible desarrollar la inteligencia sin desarrollar el lenguaje mediante el cual se comunica con sus semejantes y que se aprende en sociedad (Brotons, 2000).

Siguiendo esta filosofía, en este trabajo se presentan diferentes componentes educativos software que, bajo el epígrafe de Enclave, pretenden configurar un espacio virtual educativo, pedagógicamente diseñado para garantizar la correcta interacción de los participantes con las nuevas herramientas, así como para lograr la adecuada integración, tanto de los docentes como 
de los discentes, en un proceso formativo con una base virtual.

Así, el resto del artículo se organiza como sigue: en la segunda sección se comentan las posibilidades que ofrece y los riesgos que conlleva la aplicación de las NTIC (Nuevas Tecnologías de la Información y la Comunicación) en los procesos educativos. En la tercera sección se presenta la filosofía "Enclave", y de forma concreta algunos de los componentes educativos software que lo conforman. La sección cuatro cierra el artículo con las conclusiones del mismo.

\section{Aplicación de las nuevas tecnologías en los procesos educativos: Los espacios virtuales educativos}

El contexto en el que se desarrolla el proceso formativo a comienzos del siglo XXI es muy diferente al que se tenía diez años atrás, siendo las NTIC las responsables y las protagonistas de los cambios sufridos.

Como factores clave de estos cambios cabe citarse (Hare, 2000): el estatus de recurso activo de gran valor que toma la información; el aumento de la información en formato digital, así como de usuarios finales consumidores de dicha información; la mayor necesidad de la formación continua de los profesionales, que exige modelos de enseñanza y aprendizaje más flexibles; y el asentamiento de una cultura de la globalización, que rompe barreras físicas y temporales.

Un espacio virtual educativo efectivo debe canalizar tres vertientes básicas de las NTIC. Primeramente, la capacidad de trabajo en red, que permita una localización geográficamente dispersa de los participantes y un sincronismo o un asincronismo de las actividades formativas, según sea necesario. En segundo lugar, facilidades multimedia e hipermedia que permitan contar con información digital heterogénea y representada en diferentes formatos (texto, gráficos, sonido...) pudiéndola relacionar fácilmente. Y, por último, una interacción persona-ordenador sencilla, intuitiva, pedagógica y completa, soportada por interfaces de usuario donde se potencie la noción de usabilidad de las mismas, en lugar de buscar la espectacularidad y el encantamiento momentáneo del usuario.

La web es el ejemplo paradigmático de servicio que mejor integra los tres ejes descritos, siendo el caldo de cultivo idóneo sobre el que plantear la definición de un espaciovirtual educativo o portal educativo según la terminología web, aunque no el único.

Sin embargo, conviene advertir que en el contexto de la comunicación oral la propia biología humana deja evolutivamente dispuesto a la competencia oral en el marco social adecuado. La escritura, que aplica la capacidad gráfica humana a la comunicación simbólica, con el aprendizaje de la competencia en el uso de la interfaz lecto-escritora deja dispuesto el fundamento para convertir al sujeto en autor y productor de recursos para la comunicación, independientemente de su nivel, utilidad y calidad; desde el niño que redacta al literato que crea una obra maestra. El sistema informacional introduce un sistema de comunicación que se apoya en una teoría (la Teoría de la información), en una tecnología (las Tecnologías de la Información y la Comunicación). Por ello, el sistema de comunicación informacional plantea dos categorías de producción de objetos, que requieren competencias diferentes: la de los que son capaces de producir teoría y tecnología (de hardware y software) y la de los que poseen los conocimientos que se pueden vehicular a través del sistema informacional. Precisamente esta dicotomía de competencias es la que plantea la necesidad de reflexionar sobre el problema de las interfaces sistema informacional-usuario. Las interfaces más desarrolladas son las de ordenador-usuario, haciendo a éstos cada día más "familiares" y próximos a quienes no son ni científicos ni técnicos.

La aplicación del sistema de comunicación informacional a procesos de formación requiere de otras interfaces: las de facilitación de procesos de construcción de contenidos de formación mediante software de "segundo orden", que se convierten en facilitadores de actividad dentro del marco que posibilitan, por ejemplo, herramientas de autor de "primer orden". A interfaces 
de "segundo orden" es a las que hace referencia el contenido de este trabajo.

\section{1. Servicios de un espacio virtual educativo}

En general, un espacio virtual educativo debe ofrecer un conjunto de servicios educativos funcionales a los participantes en el proceso formativo. Éstos pueden soportar una interacción síncrona, cuando los participantes están presentes "en línea" al mismo tiempo mientras se lleva a cabo el servicio, o asíncrona, cuando la presencia de todos los participantes no es requerida para desarrollar la actividad.

Los servicios educativos pueden clasificarse diversos grupos no disjuntos entre sí (García et al., 1999):

Servicios de comunicación: Facilitan la comunicación entre los protagonistas del proceso formativo (estudiantes y profesores). En este grupo se incluyen servicios tan populares como el correo electrónico, foros de discusión (síncronos como el IRC, o asíncronos como los grupos de noticias), seminarios virtuales, videoconferencias o publicación de documentos en formato digital.

Servicios de información: Ofrecen información genérica estructurada y dispuesta de forma eficiente para un uso específico. Ejemplo de este servicio son las páginas web.

Grupos de trabajo cooperativo: Ofrecen la posibilidad de que varias personas trabajen juntas utilizando ordenadores y tecnología informática, facilitando el trabajo en equipo y un intercambio eficiente de información. Ejemplos de servicios de este grupo serían entre otros los seminarios virtuales con varios participantes activos, aplicaciones de tiempo real compartidas como escritura o dibujo cooperativos, sistemas de flujos de trabajo (workflows) o agendas comunes.

Servicios de administración: Permiten la gestión administrativa de las diversas entidades que conforman el dominio del problema del ámbito educativo, esto es, profesores, alumnos, cursos, informes estadísticos...

Servicios de entretenimiento: Son servicios, educativos o no, diseñados en su mayor medida para el ocio, como juegos en línea o tablones de noticias.

Servicios y herramientas de autor mediante las cuales los formadores pueden producir unidades de actividad que, al tiempo que recuperan los modos escritos de oferta de conocimiento, pueden incorporar el modo oral, el icónico, y el audiovisual, dotados de reticularidad, organización topológicas y navegables en función de los intereses particulares del usuario.

Estos servicios quedan establecidos en el espacio virtual educativo dentro de un conjunto de componentes software de carácter pedagógico, junto a un repositorio de información, donde quedarán almacenados los diferentes activos de información que se intercambian en el proceso educativo.

La interacción de los participantes en dicho proceso educativo se hace a través de dichos componentes software, en sus versiones cliente y servidor, donde normalmente el cliente manejado es un clásico navegador web, que da acceso al resto de los componentes.

La configuración genérica de un espacio virtual queda reflejada en la figura 1.

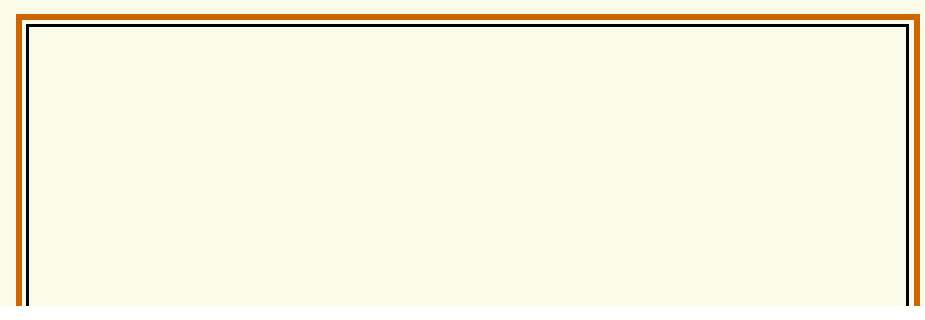




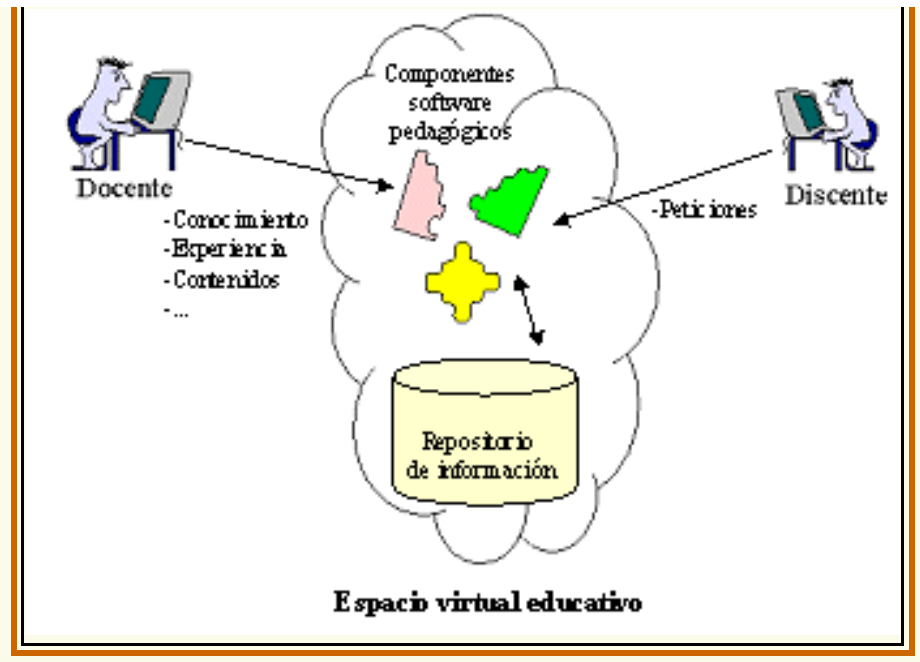

Figura 1. Configuración de un espacio virtual educativo

\section{2. Ventajas y riesgos de los espacios virtuales educativos}

La entrada en escena de los espacios educativos virtuales aporta grandes ventajas que complementan positivamente el proceso educativo tradicional. Sin embargo, el trabajo con las NTIC implica cambios en muchas áreas, y también algunos riesgos. Ambos aspectos, ventajas y riesgos, van a tratarse en este subapartado.

La introducción de las NTIC en el proceso educativo aporta los siguientes beneficios potenciales:

Descentralización del proceso educativo: El seguimiento de las clases desde lugares geográficamente distantes, ya sea de forma síncrona o asíncrona, potencia la enseñanza a distancia, las tutorías no presenciales y los seminarios virtuales.

Aumento de la calidad y la accesibilidad de los materiales didácticos: Los multimedia cambian el concepto del libro tradicional, no buscando suplantarlo sino completarlo. El texto y las fotografías se ven completadas ahora con datos en cualquier tipo de formato imaginable (vídeo, animación, sonido...) y localizados en cualquier lugar del mundo.

Procesos de aprendizajes personalizados: Al tener acceso a materiales didácticos de calidad dentro de un proceso educativo asíncrono, el alumno puede progresar según su capacidad y tiempo.

Facilidad para el trabajo colaborativo: El trabajo en equipo se ve facilitado desde el mismo instante en que mejoran y se potencian los medios de comunicación disponibles entre los participantes en una actividad docente.

Acceso universal a los recursos: Permitiendo acceso a recursos localizados de prácticamente cualquier parte del mundo.

Capacidad de adaptación o configuración del entorno de trabajo: El contexto de trabajo del usuario se adapta dependiendo de su nivel, su idioma u otras características, permitiendo un rendimiento más óptimo del interesado.

No obstante, las NTIC presentan también constricciones y riesgos más que desventajas o inconvenientes, normalmente derivados de su mala utilización, de su comprensión equivocada o de las complejidades inherentes al empleo de las NTIC como instrumento de autor. Entre aquellos riesgos cabe citar:

Desplazamiento de profesores y/o alumnos del proceso educativo: Las NTIC obligan tanto a los docentes como a los discentes a un replanteamiento de la naturaleza de la enseñanza y el aprendizaje. Los profesores, en su papel de transmisores de conocimiento, deben aprender a 
manejar las herramientas que permiten canalizar su conocimiento y experiencia en materiales didácticos asimilables por los alumnos. Los alumnos deben estar abiertos a la utilización de las NTIC y a que las referencias de consulta no se limitan a las notas de clase.

Dispersión de la información: Internet es una fuente inagotable de información, que fácilmente puede llegar a desbordar a cualquiera, y donde la calidad de los materiales accesibles es sumamente variable. Por este motivo, la mera existencia de información no basta para configurar un espacio virtual educativo (Moreno, et. al., 2000a); debe ser información contrastada, clasificada y accesible a través de los medios oportunos.

Falta de calidad en los componentes educativos software: Los servicios educativos ofertados en un espacio virtual no siempre tienen la calidad suficiente para su uso efectivo. La calidad debe mirarse desde las perspectivas técnica y pedagógica, de forma que los componentes cumplan los requisitos para los que fueron diseñados, pero además lo hagan de forma que ayuden al proceso educativo. En este aspecto los mecanismos de interacción ofrecidos por las interfaces de los componentes software son de vital importancia. Una interfaz bien diseñada debe guiar al usuario (ya sea alumno o profesor) en la actividad docente en que se halle inmerso, mientras que una interfaz incorrectamente pensada perderá al usuario en el software, y lo más grave, en el caso de los alumnos, puede inducirles a errores de concepto graves que invaliden su proceso formativo.

Precisamente, en el grupo de trabajo de los autores en la Universidad de Salamanca se está trabajando en el desarrollo de un conjunto de componentes educativos software para la definición de un espacio virtual educativo que se conoce bajo el nombre de Enclave.

En los componentes desarrollados se está tratando tanto de potenciar las ventajas aquí citadas, como combatir los riesgos expuestos. Uno de los puntos donde mayor esfuerzo se está haciendo es en la definición de los elementos de interacción entre el usuario y los componentes software, descansando la parte técnica en los especialistas del campo de la computación y la parte pedagógica en los pedagogos del grupo de trabajo. Algunos de los componentes de Enclave se presentan en el siguiente apartado.

\section{Enclave: componentes software para la definición de espacios virtuales educativos}

El espacio virtual educativo Enclave pretende ser un portal educativo configurable a las necesidades de las actividades que en él vayan a tener cabida, cuidando siempre dos elementos decisivos: la accesibilidad y la mejora del aprendizaje.

A través de los componentes desarrollados, el proceso de aprendizaje se abre a todo tipo de alumnos, desde niños a adultos de avanzada edad. La optimización del aprendizaje se logra al flexibilizar el proceso, potenciando un sistema asíncrono de estudio, salvo para actividades concretas, normalmente dirigidas a un público especializado, concreto y de avanzados conocimientos.

Actualmente existen numerosos componentes desarrollados, que han sido ampliamente probados en diferentes experiencias educativas, y otros muchos se encuentran en desarrollo. Los componentes desarrollados evolucionan a nuevas versiones, buscando la mejora continua de sus prestaciones y, por tanto, del espacio virtual educativo que conforman.

Los componentes desarrollados pueden clasificarse siguiendo diferentes patrones de categorización, sin embargo, para los fines de este artículo se van a dividir en dos grupos: componentes de autor y componentes de servicio educativo.

Los componentes de autor están orientados a que el docente genere materiales didácticos de calidad que incorporará de alguna manera al espacio virtual. Entre otros varios componentes desarrollados, cabe citar a: el gestor de actividades - destinada a la creación de unidades docentes, que incluyen problemas y su resolución; el compositor de hipertextos - herramienta destinada a crear documentos multimedia con estructura jerárquica con salida en ficheros 
XML; el generador de guías académicas (Moreno et al., 2000a) que auxilia al docente en la creación de las guías de las asignaturas que imparte.

Los componentes de servicio educativo están destinados a dar soporte a actividades docentes concretas, en las que la colaboración es una característica fundamental. Entre otros varios componentes desarrollados, cabe citar a: el seminario virtual (Moreno et al., 2000b) que permite la realización de seminarios, cuyos participantes están geográficamente distantes; el tutor de ortografía (Rodríguez Hernández et al., 2002) destinado a que los niños aprendan y corrijan sus problemas con las reglas de ortografía del español; el gestor para seguimiento de tesis, que presenta un entorno para el seguimiento de trabajos académicos por parte de un tutor (tesis, tesinas o proyectos de final de carrera).

Como muestra de los componentes desarrollados se van a presentar el compositor de hipertextos, como ejemplo de los componentes de autor, y el seminario virtual, como representante de los componentes de servicio educativo.

\section{1. El compositor de hipertextos}

Este componente está justificado por la dificultad que encuentran un gran número de docentes en crear material didáctico multimedia, principalmente por su desconocimiento de las herramientas de autor que se utilizan para estos menesteres, más pensadas para profesionales de la informática que para los expertos en otras materias.

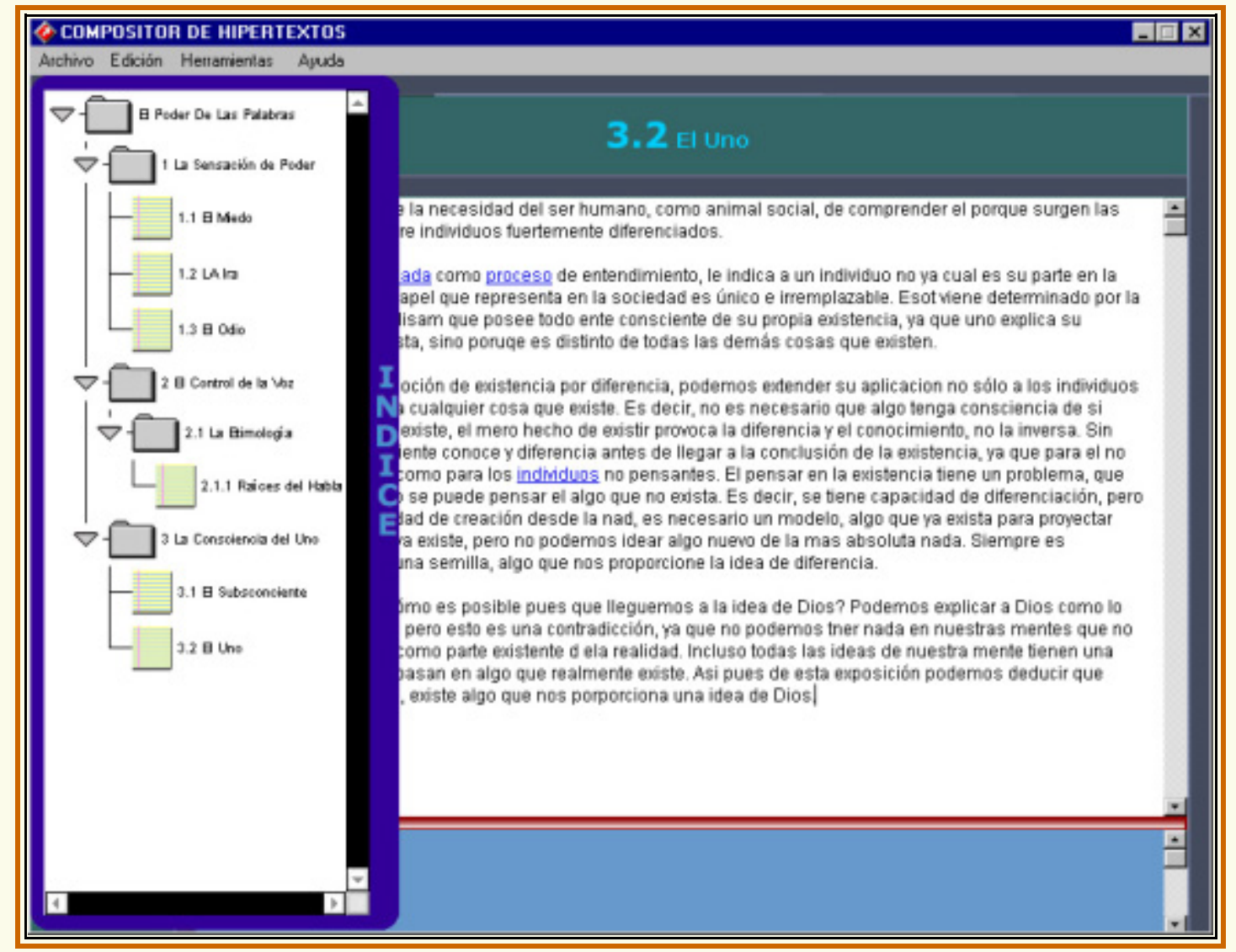

Figura 2. Interfaz básica del compositor de hipertextos

Con el mayor acceso a las nuevas tecnologías de la información, los productos multimedia se convierten en un método de aprendizaje que aportan nuevas características en la labor docente en general. Éstos alientan la exploración, la autoexpresión y un sentido de propiedad al permitir a los estudiantes manipular los elementos que conforman un documento multimedia. Además, los medios multimedia hacen el aprendizaje estimulante, atractivo y divertido.

Con la incorporación de los entornos multimedia el rol del profesor, como un mero transmisor de conocimientos, cambia hacia funciones de organización de actividades de aprendizaje, 
otorgando al alumno una mayor autonomía y responsabilidad en la búsqueda de su formación. Se consigue, además, que el alumno juegue un papel más activo en el proceso educativo propiciado por las nuevas tecnologías, viéndose más motivado a utilizar las fuentes de información disponibles, así como aquéllas que se le recomiendan. Por otra parte, estas innovaciones provocan un cambio en el concepto de aula, vista como entidad física, de manera que va cobrando un mayor sentido la noción de aula virtual, espacio que el alumno puede utilizar sin restricciones de tiempo y espacio; realidad que se hace más patente con la incorporación de las facilidades telemáticas a la educación a través de Internet y la proliferación de portales virtuales dedicados exclusivamente a la educación. Todas estas aportaciones prometen un aprendizaje más efectivo que repercutirá positivamente en la posterior actuación del alumno en la sociedad actual.

Cuando un autor se dispone a crear un texto, educativo o no, es recomendable, por no decir imprescindible, realizar una organización de los contenidos, en pro de la claridad y calidad del resultado final.

Una forma comúnmente aceptada de llevar a cabo esta organización es la realización previa de un índice de contenidos, sin límite teórico de niveles de anidamiento (aunque si práctico en aras de la claridad), de forma que obtenemos una estructura jerárquica que nos dirige en nuestro proceso creativo, el cual consistirá en ir asociando contenidos a cada entrada del índice; índice que por otra parte puede sufrir variaciones según se van dando forma a los contenidos, insertando, eliminando o cambiando entradas del mismo.

El compositor de hipertextos reproduce fielmente el proceso anteriormente explicado. La interfaz de trabajo presenta dos zonas diferenciadas y destacadas del resto, la zona de índice y la zona de trabajo, como se puede apreciar en la figura 2. Cada entrada del índice da lugar a una unidad temática o lexia que puede contener texto, material multimedia y enlaces a otras unidades o documentos.

Esta organización, además de dar lugar a una metáfora de trabajo fácilmente asimilable por cualquiera que haya escrito antes algún tipo de documento jerárquico, permite una traducción directa a un hiperdocumento bien organizado, sin más que tener en cuenta que cada lexia se corresponde con un nodo del hiperdocumento.

La equivalencia lexia-nodo es la base para el éxito del proceso de creación porque el nodo, al igual que la lexia para el autor, es una unidad de información en la que una serie de contenidos de diversa índole se combinan para transmitir una idea o concepto. El nodo es una unidad de visualización que facilitará la labor de los navegadores para mostrar el hiperdocumento y navegar a través de él. Además, esta equivalencia cumple una máxima de calidad de todo hiperdocumento, los nodos deben ser entidades autocontenidas para evitar que un concepto se divida en múltiples fragmentos, de forma que a los lectores se les facilite su reconocimiento.

El índice del documento sirve como navegador para desplazarse a las diferentes lexias de que se compone un hiperdocumento, de manera que en el modo de autor, permite acceder a la lexia en la que se quiere trabajar, o bien mediante un menú contextual hacer las modificaciones al índice que el autor estime oportuno (ver parte izquierda de la Figura 2), mientras que en el modo de lector, junto a los hipervínculos, representa la forma de navegar por el hiperdocumento.

Además, si en un momento dado tras la selección de la lexia para su lectura o modificación, el usuario no se siente a gusto con la presencia del índice, éste se puede ocultar sin más que pulsar con el ratón en la pestaña del índice, realizando la misma operación cuando se quiera que el índice vuelva a estar presente.

Un hiperdocumento complementa al texto con información de diversa índole. Un problema para el autor es mantener organizada esta información, de cara a su posible publicación en un medio ajeno a la propia herramienta de autor. 
En este sentido el compositor de hipertextos mantiene un repositorio de información vinculado a cada documento que se cree con la herramienta, de forma que se soluciona en gran parte la localización de este tipo de recursos. Pero además para su gestión por parte del autor se incorpora una serie de interfaces basadas en las metáforas de visor y formulario, y que reciben el nombre de galerías.

Una galería es un gestor de toda la información, clasificada por tipos, que el usuario está insertando en su documento, y que es almacenada en el repositorio del documento de forma totalmente transparente al usuario. Concretamente la herramienta cuenta con cuatro galerías: Galería Multimedia, Galería de Estilos, Galería de Citas y Galería de Salidas.

La Galería Multimedia proporciona el control sobre todos los elementos multimedia que tengamos insertados en el documento, con la posibilidad de reproducción. La Galería de Estilos permite dar formato al documento, eligiendo el tipo de letra, el color de los enlaces, el tipo de referencia bibliográfica o cita... La Galería de Citas permite realizar todas las operaciones relacionadas con las referencias bibliográficas en un hiperdocumento, para posteriormente generar automáticamente el apartado de referencias bibliográficas. Por último, la Galería de Salidas tiene el cometido de gestionar el formato de salida del hiperdocumento. Ya que éste se almacena en un formato propio de la herramienta, la misma proporciona salidas compatibles con otras herramientas. Se han tenido en cuenta principalmente dos ámbitos de trabajo, el relativo al textual y el de la red. Se toma como base cubrir las herramientas: Microsoft Word e Internet Explorer. Para hacer al documento compatible con Microsoft Word, el trabajo puede ser exportado a formato RTF (Rich Text Format). Para la compatibilidad con Internet Explorer se ha optado por exportar el documento en formato XML (eXtensible Markup Language) (Bray, Paoli y Sperberg-Macquenn, 2000), por su gran penetración en los últimos tiempos y por su increíble capacidad de adaptación a diferentes plataformas, ya que permiten exportar a formatos tan dispares como HTML (HyperText Markup Language) o PDF (Portable Document Format).

\section{2. El seminario virtual}

Los seminarios son actividades docentes de gran interés, pero que se ven seriamente comprometidos cuando los participantes se encuentran geográficamente distantes. Sin embargo, con una conexión a Internet y el software apropiado, organizar un seminario virtual es una tarea tan sencilla como poner de acuerdo a todos los participantes del mismo.

El software utilizado para llevar a cabo el seminario virtual se convierte así en el cuello de botella de esta actividad. Un análisis de los servicios que ofrecen los entornos de formación virtual existentes, como webCT (http://www.webct.com) o Blackboard (http://www.blackboard.com), no parecen adecuarse de forma correcta a las características propias de los seminarios. El motivo de ello puede estar en que estas herramientas no se pensaron para este fin, careciendo de la guía de un experto en el área de educación para establecer sus características funcionales básicas.

El componente desarrollado intenta solucionar las carencias encontradas en las aplicaciones estudiadas, haciendo un especial hincapié en las facilidades de moderación, prácticamente inexistentes en los servicios tipo de chat, intercambio de material (documentos, imágenes, vídeos...) y en la presencia de una pizarra virtual donde desarrollar ideas, fórmulas...

El objetivo principal de este componente es permitir la realización de seminarios entre usuarios geográficamente distantes, y no necesariamente expertos en informática, sin que esto suponga limitación alguna en el desarrollo del mismo.

Funcionalmente el componente ofrece los siguientes servicios: diálogo entre los participantes, moderación del seminario, carga y descarga de documentos, capacidad de explicación gráfica (en una pizarra) y almacenamiento de los seminarios para su consulta offline.

Todos estos servicios están accesibles a través de una única interfaz de usuario, muy intuitiva, 
que se presenta en la figura 3.

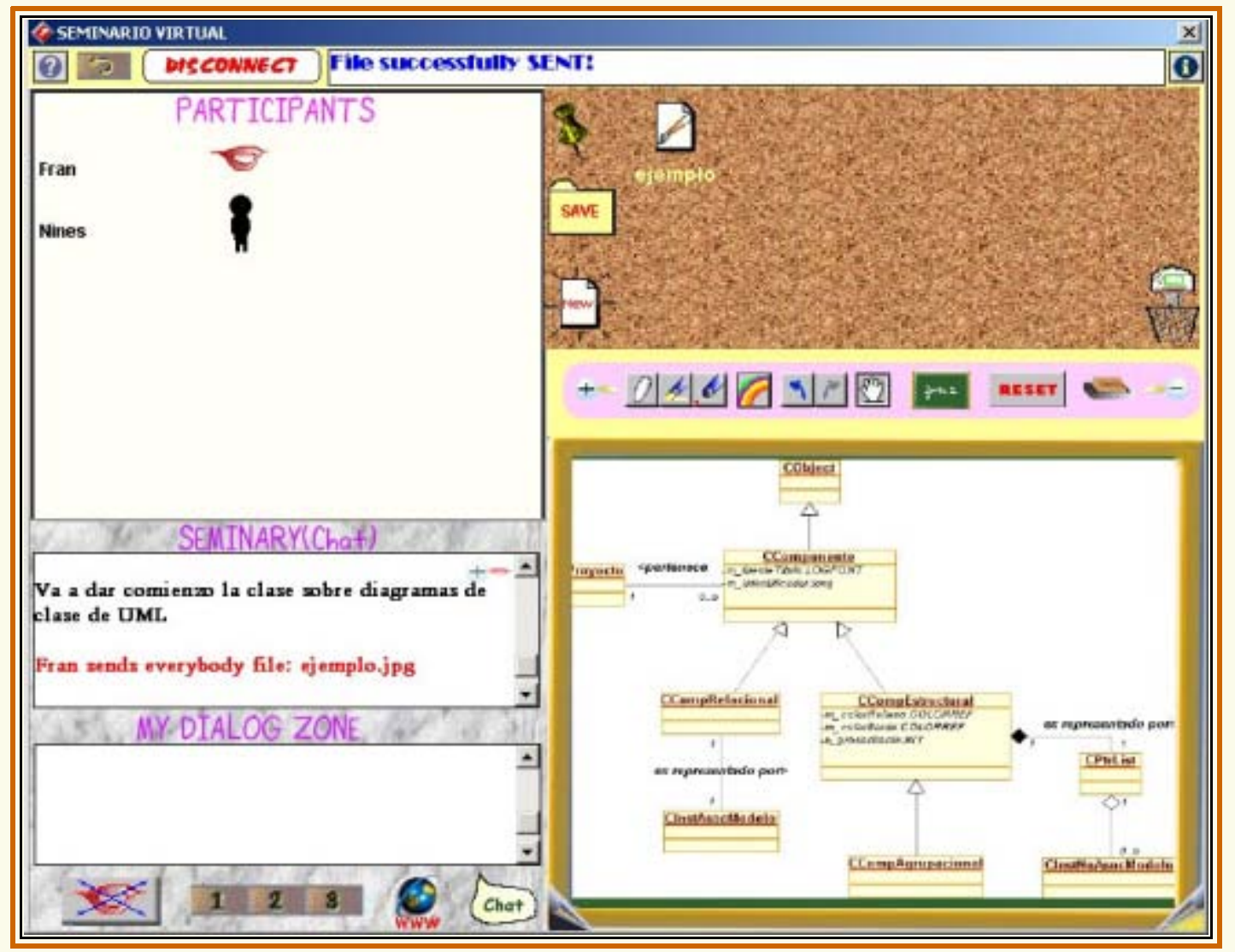

Figura 3. Interfaz del componente "seminario virtual"

A continuación se describen someramente los servicios más importantes, destacando las metáforas utilizadas para su inclusión en la interfaz de usuario.

\section{2. 1. Zona de diálogo e identificación de los participantes}

La zona de diálogo (figura 4) se corresponde con un chat clásico desde donde pueden leerse las intervenciones de los participantes y enviar las propias, así como obtener información de las incorporaciones y abandonos al seminario. En este apartado cabe destacar la metáfora de los "labios" que indica si se tiene o no la palabra. Este es un hecho importante y diferenciador, porque a diferencia de las aplicaciones de chat genéricas, donde las intervenciones pueden acabar en el caos, aquí se ha decidido moderar en todo momento las intervenciones, al igual que se haría en un seminario presencial clásico. Así, únicamente uno de los participantes poseerá el turno de palabra. Los demás pueden pedir el turno o levantar la mano para formular una pregunta.

Encima de la zona de diálogo se encuentra la zona de identificación de los participantes, que a su nombre pueden tener asignados diferentes iconos, cada uno de los cuales representa una metáfora, como se muestra en la figura 5.

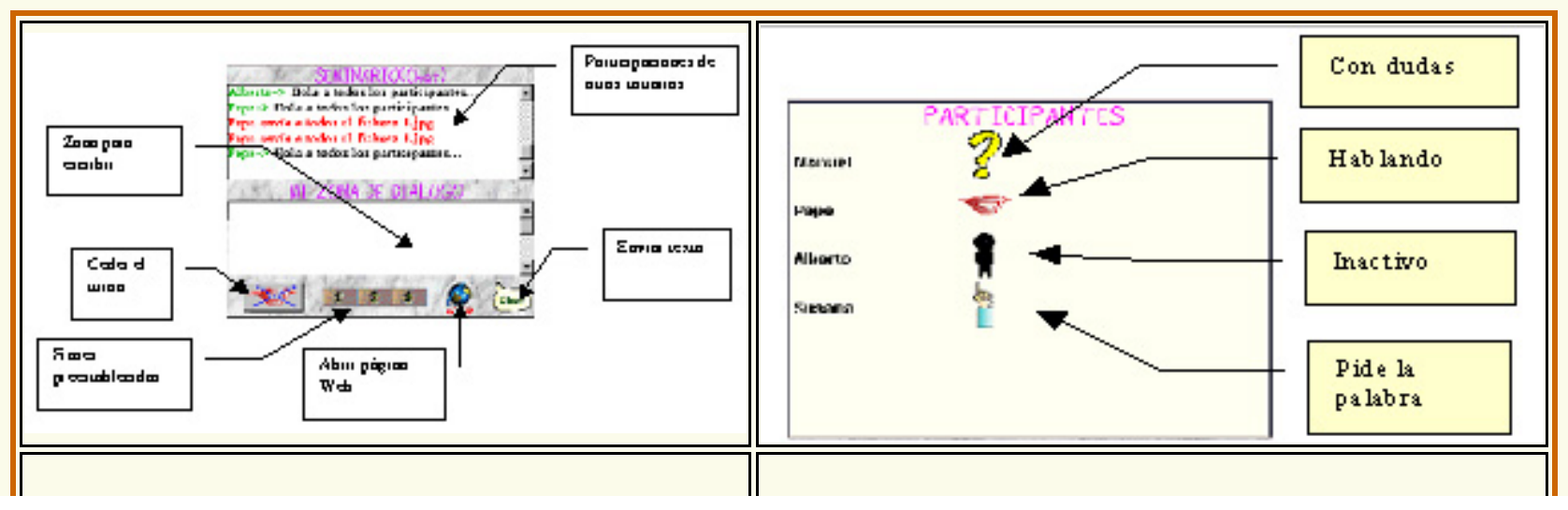




\section{2. 2. Zona de carga y descarga de documentos}

En esta zona, representada por la metáfora de un "corcho", se van a depositar todos los ficheros que cada participante en el seminario desee tener disponibles para el desarrollo del mismo (figura 6). Estos ficheros pueden ser de diferentes formatos: textos, imágenes, sonidos... Cada uno de ellos aparece representado con un tipo de icono distinto, en caso de ser de un formato desconocido se identificará por un signo de interrogación.

Además, existe una herramienta, denominada chincheta ( ) por seguir el símil del corcho, que permite enviar cualquier fichero a todos los participantes simplemente colocando la chincheta sobre él.

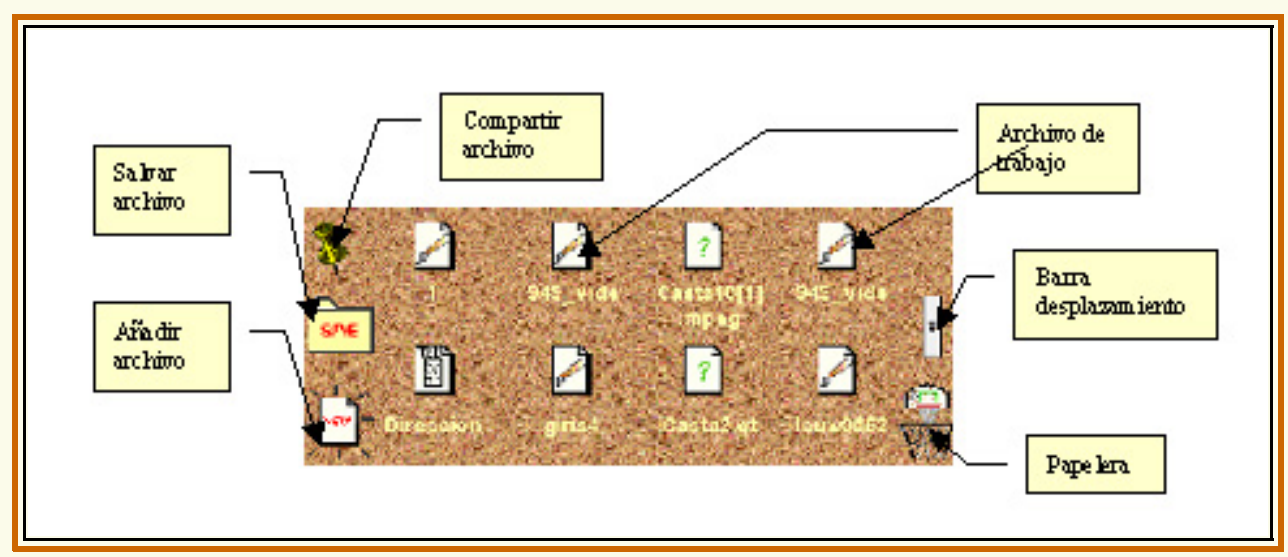

Figura 6. Zona de carga y descarga de ficheros

Otra característica interesante es que el contenido de los ficheros puede verse en la zona de explicación gráfica, sin más que arrastrar el icono del fichero sobre ella.

\section{2. 3. Zona de explicación gráfica}

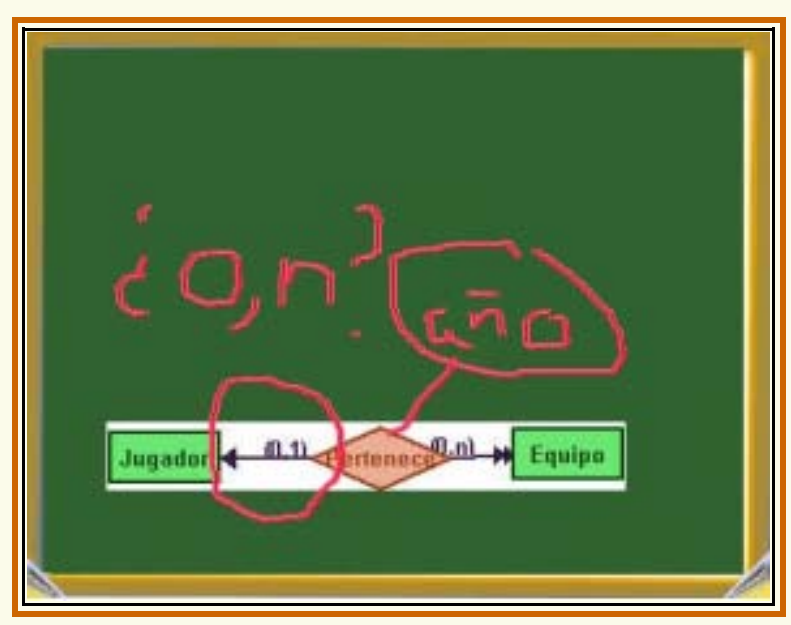

Figura 7. Pizarra

La zona de explicación gráfica está representada en la interfaz de usuario mediante la metáfora de una "pizarra" (figura 7) y constituye una parte importante del componente. Permite emular a una pizarra clásica, aunque más versátil pues es posible visualizar en ella cualquiera de los ficheros depositados en el corcho, a semejanza de un proyector de transparencias.

Esta utilidad permite apoyar las explicaciones en dibujos, fórmulas, esquemas, diagramas... 
Adicionalmente, haciendo uso de elementos tradicionales como la tiza, o más modernos como el puntero láser, se puede hacer hincapié en aquellas partes que el ponente, en posesión de la palabra, considere necesario.

La barra de herramientas que acompaña a esta zona de trabajo está compuesta por las utilidades de zoom, tiza, subrayador, puntero láser, selección de color, borrar la pizarra, entera o parcialmente, deshacer y rehacer la última acción e ir a la última pizarra. Además, se puede disponer de varias pizarras e ir pasando de una a otra. Todo lo que se vaya mostrando en ellas se visualiza para todos los participantes del seminario.

\section{Conclusiones}

En este artículo se ha expuesto la visión que tienen los autores sobre la formación virtual concretada en los espacios virtuales educativos, que si bien no deben ser sustitutos de las prácticas docentes tradicionales, pueden servir de complemento y apoyo a éstas.

En este sentido se ha presentado Enclave, un conjunto de componentes educativos software destinados a la configuración de espacios virtuales educativos pedagógicamente definidos. Dos tipos de componentes se manejan, los de autor, pensados para apoyar al docente en la creación de materiales didácticos, y los de servicios educativos, definidos para dar lugar a experiencias docentes reales.

Entre los componentes de autor se ha presentado el compositor de hipertextos, como una herramienta para la creación de materiales didácticos multimedia. Por su parte, como ejemplo de los componentes que ofrecen servicios se ha presentado el seminario virtual, donde el esfuerzo dedicado al diseño de su interfaz ha quedado patente en la facilidad de uso que han tenido sus usuarios en las diversas experiencias docentes en que se ha utilizado.

Ambos componentes tienen en común la herramienta de desarrollo, Macromedia Director. Esto surge como una restricción impuesta en un principio por el equipo de pedagogos que se incluyen en el grupo de trabajo, porque están muy acostumbrados a la forma de trabajo con productos realizados con esta herramienta. No obstante, aunque Macromedia Director da muchas facilidades para la creación de la interfaz de usuario y la obtención rápida de productos software multimedias, en los casos que se han presentado en este artículo se ha necesitado hacer un gran esfuerzo por parte del equipo de desarrollo para solventar las limitaciones que esta herramienta imponía para la consecución de una funcionalidad tan compleja como la que era requerida (comunicaciones, trabajo con ficheros, acceso a Internet...).

\section{Agradecimientos}

Este trabajo ha sido realizado conjuntamente, dentro de un ambiente de cooperación excelente, por miembros del Instituto Universitario de Ciencias de la Educación (IUCE) y del Departamento de Informática y Automática de la Universidad de Salamanca.

Este trabajo ha sido parcialmente subvencionado por la Junta de Castilla y León y la Unión Europea a través del Fondo Social Europeo mediante el proyecto de investigación SA002/01.

\section{Bibliografia}

BRAY, T.; PAOLI, J. y SPERBERG-MACQUENN, C. M. (2000) Extensible Markup Language (XML) 1.0 (Second Edition). World Wide Web Consortium Recommendation October . http://www.w3c.org/TR/2000/REC-xml-20001006.

BROTONS, J. R. (2000) Enseñanza y Nuevas Tecnologías en el Futuro, Novática,145, 30-32. GARCÍA, J. et al. (1999) Nuevas Tecnologías y Educación. PCWEEK. Editorial América Ibérica.

GIL, A. B. et al.(2001) Herramienta para al Generación de un Libro Electrónico Educativo, en RODRÍGUEZ ILLERA, L. et al. (eds.) Actas de las III Jornadas Multimedia Educativo. 
Universitat de Barcelona, pp. 277-286.

HARE, C. (2000) New Technologies and Education of Information Professionals, Teoría de la Educación. Educación y Cultura en la Sociedad de la Información, 2 . http://www3.usal.es/teoriaeducacion/

MORENO, Á. Ma . et al. (2000) Componentes Software para Entornos Virutales de Educación, en Actas de las Jornadas UNED-2000 Conocimiento, Método y Tecnologías en la Educación a Distancia. UNED, Madrid, pp. 122-126.

MORENO, A. et. al. (2000b) Seminario Virtual: llevando los espacios virtuales educativos a la práctica, en ORTEGA, M. y BRAVO, J. (2000)Actas del $2^{\circ}$ Simposio Internacional de Informática Educativa SIIE'2000. Resumen en página 74 y ponencia en versión digital - CDROM.

RODRÍGUEZ GERNÁNDEZ, R. et. al. (2002) Diseño y desarrollo de una aplicación para la enseñanza de ortografía a niños de primaria: GUIO v1.0, enActas del Congreso Interacción 2002. Universidad Carlos III.

(C) Ediciones Universidad de Salamanca

Páginas mantenidas por Francisco Ignacio Revuelta Domínguez

y Luis González Rodero

webmrte@usal.es 